\title{
Avviso ai lettori
}

L'articolo di Giovanni Pozzi che si pubblica in questo numero di Quaderni d'italianistica è un documento straordinario nato da una doppia occasione: la visita in Canada dell'insigne studioso svizzero nel marzo del 2001 e la pubblicazione, negli stessi giorni, della traduzione ungherese di Scrittrici misticbe italiane, la monumentale antologia curata insieme a Claudio Leonardi e uscita a stampa in Italia nel 1988 e di nuovo nel 1996. Giovanni Pozzi si trovava a Toronto per presentare la relazione d'apertura del convegno di St. Michael's College, Mystics, T isions, and Miracles (i cui atti sono appena stati pubblicati) e fu in quella occasione che gli chiesi un articolo per i nostri Quadermi. Rispose che l'avrebbe fatto riprendendo l'argomento del convegno e dell'opera appena riedita in traduzione. Il lavoro svolto da Padre Pozzi nell'ambito della scrittura mistica (da cui sono nate le edizioni delle opere di Maria Maddalena de' Pazzi, di Angela da Foligno e di Chiara d'Assisi, oltre all'antologia ricordata e a numerosi saggi critici) per la portata dell'ampia operazione di rivisitazione e restauro che ha coinvolto numerosi allievi e collaboratori, per la sua forza intellettuale e per l'assoluta affidabilità filologica nel costituire testi di sicuro riferimento, va senz'altro riconosciuto come fondativo di una nuova realtà critico-letteraria. E questo non solo per la sua descrizione attenta di sterminate, non comuni scritture, ma soprattutto per l'aver egli attrezzato un insieme di parametri critici di lettura e di apprezzamento di questo patrimonio letterario ancora in larga parte inesplorato. Ebbene, è proprio a integrare questo insieme di strumenti critici e di suggerimenti di lettura che si pone il notevolissimo articolo che oggi qui si pubblica. Per mia scelta personale l'articolo si riproduce esattamente come è stato inviato dall'autore, senza neanche alterare lo stile editoriale per le note e per i dettagli bibliografici.

F:G. 